\title{
Magnetic trapping of strongly-magnetized Rydberg atoms
}

\author{
J.-H. Choi, J.R. Guest ${ }^{\mathrm{a}}$, and G. Raithel ${ }^{\mathrm{b}}$ \\ FOCUS Center, Department of Physics, University of Michigan, Ann Arbor, Michigan 48109-1040, USA
}

Received 1st December 2005 / Received in final form 25 January 2006

Published online 13 April 2006 - (c) EDP Sciences, Società Italiana di Fisica, Springer-Verlag 2006

\begin{abstract}
Effective magnetic moments of drift Rydberg atoms in strong magnetic fields are obtained for different energy and angular-momentum states. Classical two-body trajectory calculations and quantummechanical one-body calculations are employed. For heavy atoms such as rubidium, the trapping dynamics can largely be explained by the net magnetic moment due to the cyclotron and the magnetron motion of the Rydberg electron. In light Rydberg atoms such as hydrogen, the intrinsic two-body nature of the dynamics becomes manifest in that the ionic motion significantly contributes to the effective magnetic moment. Also, light drift Rydberg atoms exhibit an anisotropic response to field-inhomogeneities parallel and transverse to the magnetic-field lines. The results are relevant to magnetic trapping of Rydberg atoms in strong-magnetic-field atom traps.
\end{abstract}

PACS. 32.80.Rm Multiphoton ionization and excitation to highly excited states (e.g., Rydberg states) 32.80.Pj Optical cooling of atoms; trapping - 32.10.Dk Electric and magnetic moments, polarizability 32.60.+i Zeeman and Stark effects

\section{Introduction}

Recently, cold Rydberg atoms have received considerable attention because they can be useful in quantum information processing [1], antihydrogen production [2], precision measurements [3], and in the study of complex collisioninduced dynamics [4-8]. In some proposals, the ability to trap Rydberg atoms is highly desired in order to achieve full control over both the internal and external degrees of freedom of the atoms [3,9-11]. Due to the quasi-free nature of Rydberg electrons, Rydberg atoms are highly susceptible to a variety of external fields. These large susceptibilities are the basis for Rydberg-atom-trapping methods that have been proposed [3,9-11].

The trapping of long-lived highly-excited atoms raises some issues that are distinct from those in other atomtrapping methodologies. Because of their large size, Rydberg atoms cannot be treated as point-like particles in locally constant trapping fields. The length scale over which the trapping fields vary can be of the same order as the size of the atom to be trapped. Consequently, the determination of electronic states and atom trapping potentials can become quite involved $[3,10,11]$. The situation is complicated by the high density of electronic states and trapping-field-induced couplings between those states. The combined quantum states of the center-of-mass and electronic degrees of freedom can be quite different from

\footnotetext{
a Present address: Physics Division, Argonne National Laboratory, Argonne, IL 60439, USA.

b e-mail: graithel@umich.edu
}

the field-free states $[3,10,11]$. In Rydberg-atom trapping, it also appears very important to consider how stable the trapped atoms will be against transitions into un-trapped states due to radiative interactions and couplings induced by stray electric or magnetic fields. In recent experiments, we have found that these potential difficulties can be overcome in high-magnetic-field (high- $B$ ) Rydberg-atom traps [12].

In collision-rich environments, strongly magnetized Rydberg atoms are expected to mostly reside in so-called "guiding-center" drift states [13], because there is a large density of such states and because they are long-lived. Antihydrogen Rydberg atoms prepared in nested Penning traps [14-16] presumably have been in such states. In context with the antihydrogen work, it has recently been shown that drift-state Rydberg atoms can be trapped in homogeneous magnetic and inhomogeneous electric fields $[17,18]$. Due to their electric polarizability, driftstate Rydberg atoms are attracted to regions of large electric fields. In the present paper, we theoretically investigate the magnetic trapping of drift-state Rydberg atoms in inhomogeneous magnetic fields. We cover the trapping of both heavy (rubidium) and light (hydrogen) Rydberg atoms in high- $B$ traps. The methods we utilize are twobody classical trajectory calculations in strong, inhomogeneous $B$-fields and one-body quantum-mechanical calculations. The quantum calculations describe the case of infinite nuclear mass, which usually is a good approximation for heavy atoms such as rubidium. We apply the obtained results in order to determine the character of 
the $\mathrm{Rb}$ Rydberg atoms that have recently been trapped in a high- $B$ atom trap [12]. The results obtained on the trapping of $\mathrm{H}$ Rydberg atoms may become useful in antihydrogen research.

\section{Trapping drift Rydberg atoms}

\subsection{Review of drift Rydberg atoms}

In strongly magnetized Rydberg atoms, the dominant interaction of the Rydberg electron often is the interaction with the external magnetic field. In the guiding-center drift Rydberg atoms that we study in this paper, this is always the case. Classically, the motion of the Rydberg electron in these atoms is characterized by a fast, smalldiameter cyclotron oscillation, a bounce motion parallel to the magnetic-field lines (" $z$-bounce" motion), and a slow $\mathbf{E} \times \mathbf{B}$ magnetron drift of the electron in the plane transverse to $\mathbf{B}$. The $z$-bounce motion is driven by the component of the atomic Coulomb electric field parallel to $\mathbf{B}$, while the magnetron drift results from the electricfield component transverse to $\mathbf{B}$. Due to the different time scales of these three components of the Rydberg-electron motion, the drift Rydberg states are stable [13] and can be quantized using semiclassical quantization rules [19]. The cyclotron component of the motion generates a level structure similar to that of free-electron Landau levels [20], while the $z$-bounce motion generates Rydberg-like level series below each Landau level $[19,21,22]$. This quantization structure entails multiple ionization potentials and a complex electric-field ionization behavior [23]. The finest quantization structure of drift-state atoms is due to the slow magnetron motion and becomes manifest when solving the problem for different values of the conserved magnetic quantum number $m$ [22].

Following the outlined hierarchy of interactions, the dominant interaction of a drift-state Rydberg electron is that with the magnetic field. If the magnetic field is inhomogeneous, the guiding center of the quasi-free Rydberg electron will experience forces similar to those acting on free electrons gyrating in the same field. A strategy to trap drift-state Rydberg atoms therefore is to apply an inhomogeneous magnetic field suited to trap the quasifree Rydberg electron. The entire atom may then become trapped because of the weak residual interatomic Coulomb force that binds the atomic core to the trapped electron.

While this approach is oversimplified, we can use it to point out a few fundamental differences between the magnetic trapping of ground-state atoms and atoms in drift Rydberg states. Magnetic trapping of ground-state atoms is based on "adiabatic following" of the atomic magnetic moment, making it possible to derive the atomic centerof-mass dynamics from a conservative trapping potential $V(\mathbf{R})=-\mu_{z} B(\mathbf{R})$, where $\mathbf{R}$ is the atomic center-of-mass coordinate and $\mu_{z}$ is the (fixed) projection of the magnetic moment onto the local magnetic-field direction. In contrast, the trapping of free electrons in suitable inhomogeneous magnetic fields exploits a combination of magnetic bottle forces, which act in the magnetic-field direction, (a) Rubidium

(b) Hydrogen

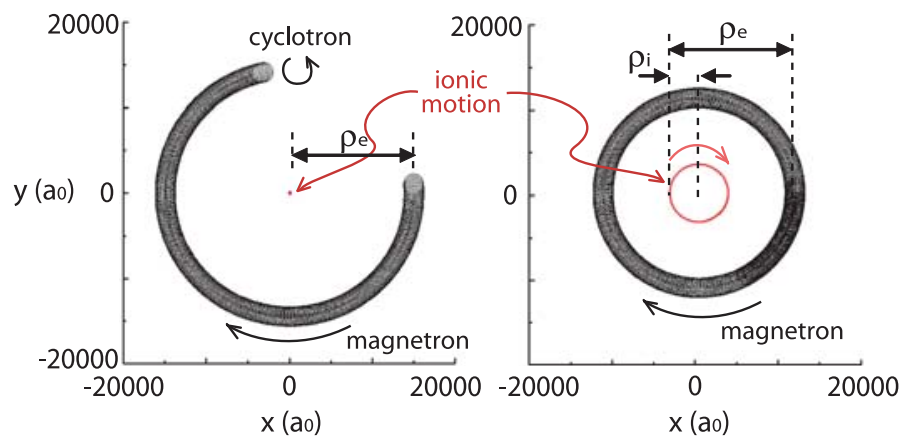

Fig. 1. Electron and ion trajectories in (a) rubidium and (b) hydrogen drift Rydberg atoms at $3 \mathrm{~T}$ field integrated over 400 cyclotron periods of the electron. The magnetic field is perpendicular to the $x y$-plane. In both cases, the magnetron frequency of the electron and the orbital frequency of the ion are the same, and $\rho_{\mathrm{e}} \approx 15000 . \rho_{\mathrm{e}}$ and $\rho_{\mathrm{i}}$ are related by equation (3). In the hydrogen case, $\rho_{\mathrm{i}} \approx 3500$.

and gradient and curvature drift motions transverse to $\mathbf{B}$. As outlined in reference [24], the magnetic-bottle effect can be formulated using a one-dimensional conservative potential $V(\eta)=-\mu_{z} B(\eta)$, where $\eta$ is a curved coordinate along the magnetic field lines and $\mu_{z}$ the adiabatically conserved magnetic moment of the cyclotron motion (which always points in the $-\hat{\eta}$ direction). However, the free-electron drift motions transverse to $\mathbf{B}$ cannot be described by a potential formulation. Thus, magnetic forces on the center-of-mass of weakly bound Rydberg atoms in strong, inhomogeneous magnetic fields may not always be derivable from conservative potentials. Also, one should expect the response of such atoms to field inhomogeneities to be anisotropic.

\subsection{Simple models of the magnetic moment of drift Rydberg atoms}

The (classical) motion of the Rydberg electron in drift states, shown in Figure 1, can be decomposed into a fast cyclotron motion, an intermediate bounce motion parallel to the magnetic field lines, and a slow magnetron drift $[19,21,22]$. Both the cyclotron and magnetron motion are equivalent to circular currents and therefore contribute to the net magnetic moment of the Rydberg atom. Thereby, the average electric field that gives rise to the magnetron motion depends on the bounce motion, and so does the magnetron magnetic moment. A third contribution to the magnetic moment arises from the ionic motion, which in bound atoms is a circular motion in the plane transverse to $\mathbf{B}$ with a frequency identical to that of the electron magnetron motion. In the following, expressions for the net magnetic moment of stable two-particle drift atoms are provided.

Regardless of the mass of the ionic particle, the cyclotron motion of the electron (smallest circular motion in Fig. 1) produces a magnetic moment, in units of the 
Bohr magneton $\mu_{\mathrm{B}}$, of

$$
\mu_{\mathrm{cyc}}=-2 E_{\mathrm{cyc}} / B
$$

where $E_{\text {cyc }}$ is the cyclotron energy in atomic units and $B$ the magnetic field in atomic units. The magnetron motion of the electron, i.e. the $\mathbf{E} \times \mathbf{B}$-drift motion of the electron in the Coulomb field of the ion, produces a magnetic moment opposite to that of the cyclotron moment (large circular motions in Fig. 1). The magnetron magnetic moment, in the unit of Bohr magneton, is given by

$$
\mu_{\text {mag }}=+\left\langle F_{\text {trans }}\right\rangle\left(\rho_{\mathrm{e}}-\rho_{\mathrm{i}}\right) / B
$$

where $\left(\rho_{\mathrm{e}}-\rho_{\mathrm{i}}\right)$ is the absolute radius of the electron magnetron motion, and $\left\langle F_{\text {trans }}\right\rangle$ is the transverse component of the atomic Coulomb field averaged over one period of the $z$-bounce motion. The magnetic moment due to the ionic motion depends on the absolute radius of the ion motion, $\rho_{\mathrm{i}}$ (see Fig. 1). Lorentz and Coulomb force act in the same direction and generate the centripetal force on the ion orbit. The orbital frequency of the ion equals the magnetron frequency of the electron, which is $\omega=\left\langle F_{\text {trans }}\right\rangle /\left[B\left(\rho_{\mathrm{e}}-\rho_{\mathrm{i}}\right)\right]$. One finds that

$$
\rho_{\mathrm{i}}=\frac{\rho_{\mathrm{e}}^{2}}{\rho_{\mathrm{e}}+M_{\mathrm{i}}\left\langle F_{\text {trans }}\right\rangle / B^{2}},
$$

where $M_{\mathrm{i}}$ is the ion mass, and

$$
\mu_{\text {ion }}=\rho_{\mathrm{i}}^{2}\left\langle F_{\text {trans }}\right\rangle /\left(\rho_{\mathrm{e}}-\rho_{\mathrm{i}}\right) .
$$

For planar electron motion, the electric field $\left\langle F_{\text {trans }}\right\rangle=$ $1 / \rho_{\mathrm{e}}^{2}$. For non-planar motion, $\left\langle F_{\text {trans }}\right\rangle$ is obtained using the inverse of the velocity $v_{\|}$of the electron parallel to $B$ as a weighting factor, $P(z)=1 / \sqrt{2\left(E_{||}+1 / \sqrt{\rho_{\mathrm{e}}^{2}+z^{2}}\right)}$. There, $E_{\| \mid}$is the total energy of the $z$-bounce motion. With the amplitude of the $z$-bounce motion, $z_{0}=\sqrt{E_{\|}^{-2}-\rho_{\mathrm{e}}^{2}}$, we have

$$
\left\langle F_{\text {trans }}\right\rangle=\frac{\int_{-z_{0}}^{z_{0}} \frac{\rho_{\mathrm{e}}}{\sqrt{z^{2}+\rho_{\mathrm{e}}^{2}}} P(z) d z}{\int_{-z_{0}}^{z_{0}} P(z) d z} .
$$

The following equations (6-9) provide the net magnetic moment, $\mu=\mu_{\mathrm{cyc}}+\mu_{\mathrm{mag}}+\mu_{\mathrm{ion}}$, for four cases of particular interest. In the most general case when the ion mass $M_{\mathrm{i}}$ is finite and the electronic motion is non-planar,

$$
\mu_{\mathrm{M}_{\mathrm{i}}}=-\frac{2 E_{\mathrm{cyc}}}{B}+\frac{\left\langle F_{\text {trans }}\right\rangle\left(\rho_{\mathrm{e}}-\rho_{\mathrm{i}}\right)}{B}-\frac{\rho_{\mathrm{i}}^{2}\left\langle F_{\text {trans }}\right\rangle}{B\left(\rho_{\mathrm{e}}-\rho_{\mathrm{i}}\right)}
$$

with $\rho_{\mathrm{i}}$ given by equation (3). For the case of planar motion, the results reduce to $\rho_{\mathrm{i}}=\rho_{\mathrm{e}}^{2} /\left[\rho_{\mathrm{e}}+M / \rho_{\mathrm{e}}^{2} B^{2}\right]$ and

$$
\mu_{\mathrm{M}_{\mathrm{i}}, \text { planar }}=-\frac{2 E_{\mathrm{cyc}}}{B}+\frac{\rho_{\mathrm{e}}-\rho_{\mathrm{i}}}{B \rho_{\mathrm{e}}^{2}}-\frac{\rho_{\mathrm{i}}^{2}}{B \rho_{\mathrm{e}}^{2}\left(\rho_{\mathrm{e}}-\rho_{\mathrm{i}}\right)} .
$$

For the case of infinite ion mass and non-planar motion, the results reduce to $\rho_{\mathrm{i}}=0$ and

$$
\mu_{\mathrm{M}_{\mathrm{i}}=\infty}=-\frac{2 E_{\text {cyc }}}{B}+\frac{\left\langle F_{\text {trans }}\right\rangle \rho_{\mathrm{e}}}{B} .
$$

Finally, for the simplest case of infinite ion mass and planar motion it is $\rho_{\mathrm{i}}=0$ and

$$
\mu_{\mathrm{M}_{\mathrm{i}}=\infty, \text { planar }}=-\frac{2 E_{\mathrm{cyc}}}{B}+\frac{1}{B \rho_{\mathrm{e}}} .
$$

As we will find, these moments describe magnetic forces quite well for the case that the field inhomogeneity is parallel to $\mathbf{B}$, i.e. $\nabla|\mathbf{B}| \mid \mathbf{B}$. This is not surprising because for guiding-center drift orbits such as depicted in Figure 1 all three motions that contribute to the dipole moment - cyclotron, magnetron, ion motion - are circular, and the magnetic force simply reflects the non-zero projection of the respective Lorentz forces onto the magneticfield directions at the centers of the respective circles. Low-magnetic-field-seeking character, which is required for atom trapping, results from the negative contributions to the magnetic moment (cyclotron motion of the electron and motion of the ion), while the magnetron motion of the electron produces high-field-seeking character. Therefore, details of the motion (namely $E_{\mathrm{cyc}}, E_{\|}, \rho_{\mathrm{e}}$ and $M_{\mathrm{i}}$ ) determine whether an atom can be magnetically trapped or not.

Magnetic forces that result from field inhomogeneities transverse to $\mathbf{B}$, i.e. $\nabla|\mathbf{B}| \perp \mathbf{B}$, cannot be derived in a straightforward manner. For atoms in low-lying states, which merely experience linear Zeeman shifts at the field strengths of interest, this case can usually be treated by assuming that the net magnetic moment is fixed while the atom moves in the field, and that the magnetic moment adiabatically follows the $\mathbf{B}$-direction (i.e. the component of the magnetic moment in $\mathbf{B}$-direction is fixed). These assumptions do not always hold for weakly bound two-body systems in strong magnetic fields.

It is further noted that many combinations of the parameters $\left(E_{\mathrm{cyc}}, E_{\|}, \rho_{\mathrm{e}}\right.$ and $\left.M_{\mathrm{i}}\right)$ will not yield regular orbits such as in Figure 1. For irregular orbits, the above equations for the magnetic moment will not apply. For an orbit to be stable, the variation of the electric field sampled by the cyclotron motion must be moderate, i.e. the radius of the cyclotron motion must be much less than the radius of the magnetron motion. This condition requires $\rho_{\mathrm{e}} \gg$ $\sqrt{2 E_{\mathrm{cyc}} / B^{2}}$. For the case $B=3 \mathrm{~T}$ and $E_{\|}=1.2 \times 10^{-4}$, discussed in Section 3, it must be $\rho_{\mathrm{e}} \gg 1200$. Further, for an orbit to be stable, the frequencies of the cyclotron, $z$-bounce and magnetron motions, denoted $\omega_{\text {cyc }}, \omega_{z}$ and $\omega_{\text {mag }}$, respectively, must differ by significant factors (so that the three motions adiabatically separate). For small $\rho_{\mathrm{e}}$, where this condition is at risk of being violated, it is $\omega_{\text {cyc }} / \omega_{z} \approx \omega_{z} / \omega_{\text {mag }} \approx B \rho_{\mathrm{e}}^{3 / 2}$. Thus, the stability conditions $\omega_{\text {cyc }} / \omega_{z} \gg 1$ and $\omega_{z} / \omega_{\text {mag }} \gg 1$ are satisfied by requiring $\rho_{\mathrm{e}} \gg B^{-2 / 3}\left(\rho_{\mathrm{e}} \gg 1800\right.$ for $\left.B=3 \mathrm{~T}\right)$. Another stability requirement for regular two-body drift trajectories is that there must be no (near-)resonant energy transfer between electron and ion motion. Therefore, the cyclotron frequency of the ion, $\omega_{\mathrm{cyc}, \mathrm{i}}=B / M_{\mathrm{i}}$, which is the highest frequency of the ionic motion, must be much less than the magnetron frequency of the electron, $\omega_{\mathrm{mag}}$, which is the lowest frequency of the electronic motion. For planar orbits, $\omega_{\text {mag }}=1 /\left[B \rho_{\mathrm{e}}^{2}\left(\rho_{\mathrm{e}}-\rho_{\mathrm{i}}\right)\right]$, with $\rho_{\mathrm{i}}$ given by 
equation (3). Hydrogen drift atoms in $B=3 \mathrm{~T}$ fields, discussed in Section 3, are found to be stable with regard to this stability condition if $\rho_{\mathrm{e}} \lesssim 20000$. For rubidium atoms in $B=3 \mathrm{~T}$ fields, this stability condition requires $\rho_{\mathrm{e}} \lesssim 100000$, which is obviously much less restrictive than in the case of hydrogen. In Section 3 we provide results in the range $3000 \leq \rho_{\mathrm{e}} \leq 20000$, in which stable drift trajectories for both rubidium and hydrogen atoms in 3 Tesla magnetic fields exist.

\section{Classical trajectory calculations}

\subsection{Computational method}

The uncertainties expressed in the cautioning remarks made in the previous paragraphs have motivated us to derive magnetic forces and effective magnetic moments from exact trajectory calculations. Since even in a homogeneous magnetic field the relative and the center-of-mass motion do not separate [25], all trajectory calculations we have performed are two-body calculations. Test atoms with well-defined initial conditions for electron and ion phase-space coordinates are immersed into a strong, moderately inhomogeneous magnetic field, and the equations of motion of the two-body problem are numerically solved using the Runge-Kutta method. It is found that in response to the field inhomogeneity the center-of-mass positions of the test atoms gradually accelerate over thousands of cyclotron periods. The observed center-of-mass accelerations are employed to establish relationships between the center-of-mass force $\mathbf{F}$ and the field inhomogeneity of the form

$$
\mathbf{F}=\mu_{z}[(\nabla|\mathbf{B}|) \cdot \hat{\mathbf{z}}] \hat{\mathbf{z}}+\mu_{\rho}[(\nabla|\mathbf{B}|) \cdot \hat{\rho}] \hat{\rho}
$$

where $\mu_{z}$ and $\mu_{\rho}$ are effective longitudinal and transverse moments, $\hat{\mathbf{z}}$ is a unit vector in $\mathbf{B}$-direction, and $\hat{\rho}$ a unit vector in the direction of the $\nabla|\mathbf{B}|$-component that is transverse to $\hat{\mathbf{z}}$ (i.e. for the case $\hat{\mathbf{z}} \times(\nabla|\mathbf{B}|) \neq 0$ it is $\hat{\rho}=(\hat{\mathbf{z}} \times(\nabla|\mathbf{B}|)) \times \hat{\mathbf{z}} /|\hat{\mathbf{z}} \times(\nabla|\mathbf{B}|)|$, while for the case $\hat{\mathbf{z}} \times(\nabla|\mathbf{B}|)=0$ there is no $\hat{\rho}$-component in equation (10)). Relationships such as described in equation (10) are found to apply as long as the atom remains bound. Thereby, atoms are considered bound if both particles remain in the close vicinity of a common center-of-mass. In the case of bound systems, the center-of-mass gradually accelerates - under the influence of magnetic forces - on a time scale that is slow compared with all internal time scales of the atom. In this paper, we are only interested in the properties of bound systems. We find that the effective moments $\mu_{z}$ and $\mu_{\rho}$ of bound atoms are different, in general. For ionized atoms, i.e. if both particles are performing unrelated drift motions in the inhomogeneous magnetic field, the center-of-mass dynamics is dominated by the motion of the heavy particle, whose gradient and curvature drift obviously cannot be described with equation (10) at all.

The inhomogeneous magnetic field used in our calculations is $\mathbf{B}=\hat{\mathbf{x}}(\beta x-\alpha x z)+\hat{\mathbf{y}}(-\beta y-\alpha y z)+\hat{\mathbf{z}}\left[B_{0}+\right.$ $\left.\alpha z^{2}-(\alpha / 2)\left(x^{2}+y^{2}\right)\right]$, and the initial location of the atom is typically chosen near the origin. Typically, we use a bias magnetic field at the trap center of $B_{0}=3 \mathrm{~T}$. To simulate magnetic trapping in a Ioffe-Pritchard trap, we have used a curvature parameter of $\alpha=10^{6} \mathrm{~T} / \mathrm{m}^{2}$ and a transverse field gradient of $\beta=3000 \mathrm{~T} / \mathrm{m}$. These values are sufficiently small that the magnetic field does not significantly vary over the atomic size (= electron-ion separation), and they are large enough that the accelerations acting on the atoms can be reliably determined within reasonably short computation times (about $10^{6}$ cyclotron periods). It is noted that values of $\alpha$ and $\beta$ used in the calculations are about three orders of magnitude larger than what could be realized in experiments [12].

In a homogeneous $B$-field there exists a constant of motion called the pseudomomentum [25]

$$
\mathbf{K}=\Pi+\mathbf{B} \times\left(\mathbf{r}_{\mathrm{e}}-\mathbf{r}_{\mathrm{i}}\right),
$$

where the kinetic center-of-mass momentum $\Pi$ and the center-of-mass position $\mathbf{R}$ are defined as $\sum_{k=\mathrm{e}, \mathrm{i}} M_{k} \dot{\mathbf{r}}_{k}$ and $\left(\sum_{k=\mathrm{e}, \mathrm{i}} M_{k} \mathbf{r}_{k}\right) /\left(\sum_{k=\mathrm{e}, \mathrm{i}} M_{k}\right)$, respectively. The subscripts $e$ and $i$ identify electronic and ionic particle coordinates $\mathbf{r}$ and masses $M$, respectively. The initial conditions used in our calculations were for $\mathbf{K}=0$, corresponding to Rydberg atoms that are initially stationary (on a time scale sufficiently long to average over all internal motions of the atoms).

The initial locations of the test atoms are chosen at distances of about $10^{6}$ atomic units from the origin. Depending on whether the initial displacement is in radial $(x$ - or $y$-) or in $z$-direction, and depending on the sign of the effective magnetic moment, the center-of-mass trajectory of the atom is magnetically trapped or anti-trapped. The effective magnetic moment follows from respective fits $R(t)=R_{0} \cos (\omega t)$ or $R(t)=R_{0} \cosh (\omega t)$ to the centerof-mass trajectory of the atom. There, $R_{0}$ is the initial center-of-mass displacement in the coordinate direction of interest. For initial displacement in $z$-direction, it is $\nabla|\mathbf{B}| \| \mathbf{B}$, and the effective magnetic moment $\mu_{z}$ is obtained from the fit parameter $\omega$ using $\omega=\sqrt{\left|2 \mu_{z} \alpha / M\right|}$. For initial displacement in radial direction, it is $\nabla|\mathbf{B}| \perp \mathbf{B}$, and the effective magnetic moment $\mu_{\rho}$ is obtained using $\omega=\sqrt{\left|\left(\beta^{2}-B_{0} \alpha\right) \mu_{\rho} / B_{0} M\right|}$. The signs of the effective moments follow from the magnetic-field parameters and the type of the observed center-of-mass trajectory (magnetically trapped or anti-trapped).

\subsection{Results of trajectory calculations}

\subsubsection{Rubidium Rydberg atoms}

Effective magnetic moments $\mu_{z}$ and $\mu_{\rho}$ of rubidium drift Rydberg atoms obtained from trajectory calculations at $B_{0}=3 \mathrm{~T}, \alpha=10^{6} \mathrm{~T} / \mathrm{m}^{2}$ and $\beta=0$ are plotted vs. $\rho_{\mathrm{e}}$ in Figure 2. For initial conditions $E_{\text {cyc }}, E_{\|}, \rho_{\mathrm{e}}$ and $M_{\mathrm{i}}$ that yield regular drift orbits, we generally observe that equation (10) describes the center-of-mass motion very well. We note that the variation of $\rho_{\mathrm{e}}$ amounts to a variation of the magnetic quantum number $m$, which for the atoms studied in this paper is negative [26] and has magnitudes 

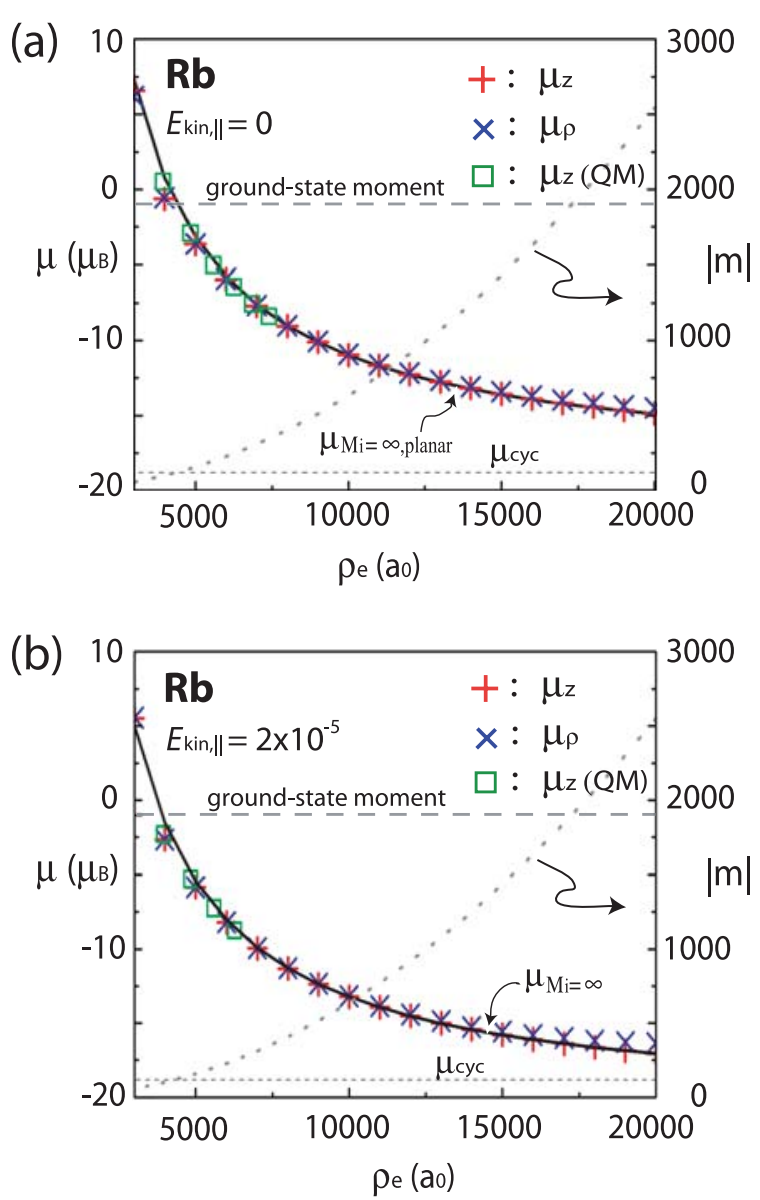

Fig. 2. Effective magnetic moments $\mu_{z}$ and $\mu_{\rho}$, as defined in equation (10), of Rb drift Rydberg atoms as a function of the guiding-center position $\rho_{\mathrm{e}}$. The magnetic moments are obtained from classical two-body trajectory calculations in a strong, inhomogeneous magnetic field of about $3 \mathrm{~T}$. The kinetic energy of the motion perpendicular to B-field is $E_{\text {cyc }}=$ $1.2 \times 10^{-4}$. The initial kinetic energy parallel to the $\mathbf{B}$-field is $E_{\text {kin, } \|}=0$ in (a) and $2 \times 10^{-5}$ in (b). The solid lines show magnetic moments obtained from equation (9) in panel (a) and equation (8) in panel (b). As a reference, the magnetic moments of a free electron, $\mu_{\text {cyc }}=-2 E_{\text {cyc }} / B$ (lines at $\mu \approx-18.5 \mu_{\mathrm{B}}$ ) and of a ground-state $\mathrm{Rb}$ atom (lines at $\left.\mu_{z}=-\mu_{\mathrm{B}}\right)$ are also shown. The squares show magnetic moments obtained from a quantum-mechanical calculation (see Fig. 4). The dotted line represents the relationship between $|m|$ and $\rho_{\mathrm{e}}$ (right axis). A colour version of the figure is available in electronic form at http://www.eurphysj.org.

as indicated on the right axes of Figures 2 and 3. Since the motion of the orbits used in Figure $2 \mathrm{a}$ is confined to the transverse plane $(\perp \mathbf{B})$, and because $M_{\mathrm{i}} \approx \infty$ for rubidium, equation (9) approximates the numerically obtained values for $\mu_{z}$ very well. Furthermore, $\mu_{z}$ and $\mu_{\rho}$ are found to be almost identical.

When Rydberg electrons are allowed to bounce along the magnetic field lines, equation (10) still provides a valid description of the center-of-mass motion. However, the average transverse electric field acting on the Rydberg elec-

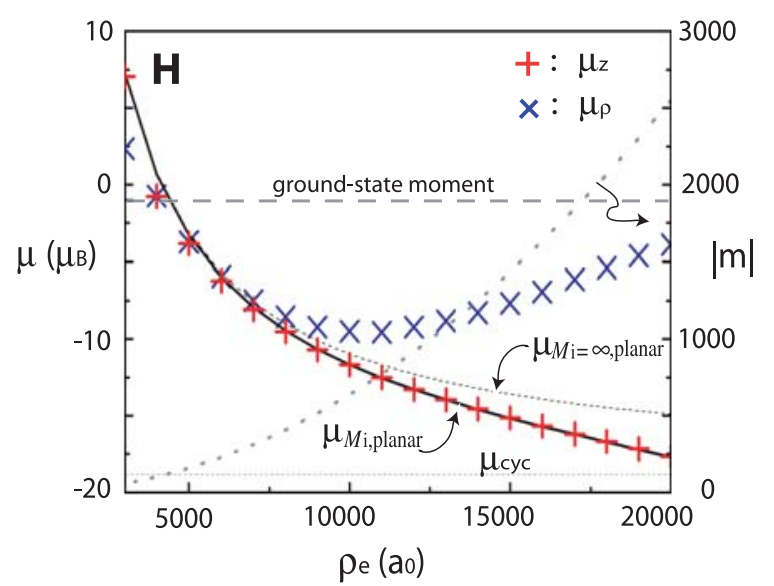

Fig. 3. Magnetic moments of hydrogen drift Rydberg atoms for transverse energy $E_{\text {cyc }}=1.2 \times 10^{-4}$ and longitudinal energy $E_{\text {kin, } \|}=0$ vs. the guiding-center position $\rho_{\mathrm{e}}$. The magnetic moments are obtained from classical two-body trajectory calculations in a strong, inhomogeneous magnetic field of about $3 \mathrm{~T}$. The calculated values of $\mu_{z}$ agree well with equation (7) (solid line). For large values of $\rho_{\mathrm{e}}$ (or $\left.|m|\right), \mu_{z}$ and $\mu_{\rho}$ are significantly different, indicating a highly anisotropic response of the atom to magnetic-field inhomogeneities. A colour version of the figure is available in electronic form at http://www . eurphysj.org.

tron $\left(\left\langle F_{\text {trans }}\right\rangle\right.$ in Eq. $\left.(5)\right)$ diminishes because the average distance between the Rydberg electron and the ion increases, and because only the component of the Coulomb field transverse to $\mathbf{B}$ is taken. Due to the diminished transverse electric field, both the magnetron velocity and the magnetron magnetic moment become reduced, and the atoms become more low-field-seeking, i.e. they have magnetic moments that are more negative than in the case of planar motion. This becomes evident by comparing the moments in Figure 2b, which have been obtained for a (total) bounce energy of $E_{\|}=2 \times 10^{-5}$, with those in Figure $2 \mathrm{a}$. For large values of $\rho_{\mathrm{e}}$, a slight difference between $\mu_{z}$ and $\mu_{\rho}$ becomes noticeable. Further, we find that equation (8) approximates the numerically obtained values for $\mu_{z}$ in Figure $2 \mathrm{~b}$ very well.

\subsubsection{Hydrogen Rydberg atoms}

Effective moments $\mu_{z}$ and $\mu_{\rho}$ of hydrogen drift Rydberg atoms are displayed in Figure 3. The figure shows that equation (7), represented by the solid line, provides a valid approximation to $\mu_{z}$, except for small $\rho_{\mathrm{e}}$. For small $\rho_{\mathrm{e}}$, equation (7) gradually breaks down because with decreasing $\rho_{\mathrm{e}}$ the time scales of the cyclotron, $z$-bounce and magnetron components of the electronic motion become increasingly similar, and orbits tend to turn from regular drift orbits into chaotic orbits (see discussion at the end of Sect. 2.2). Comparing the moments obtained from equation (7) (solid line in Fig. 3) and (9) (short-dashed line in Fig. 3), it is further noted that the value of $\mu_{z}$ for hydrogen is more negative than that for heavy ionic masses. 
Thus, in the coordinate direction parallel to $\mathbf{B}$ hydrogen drift-state Rydberg atoms are more easily trapped than heavy ones. This difference is due to two effects. First, in the case of hydrogen the ionic motion contributes a significant negative amount to $\mu_{z}$ (which is absent in the case of infinite ion mass). Second, in the case of hydrogen the positive contribution of the electron magnetron motion to $\mu_{z}$ is reduced relative to that in the case of infinite ion mass. The latter difference is due to the dependence of the electron magnetron radius, $\rho_{\mathrm{e}}-\rho_{\mathrm{i}}$, on the ion mass (see Fig. 1).

Figure 3 further shows that in the case of hydrogen the difference between $\mu_{z}$ and $\mu_{\rho}$ becomes a dominant effect. With increasing value of $\rho_{\mathrm{e}}$, the atomic binding of the drift atoms decreases, and $\left|\mu_{\rho}\right|$ is seen to become considerably less than $\left|\mu_{z}\right|$. Thus, with increasing $\rho_{\mathrm{e}}$ radial trapping becomes considerably less effective than longitudinal trapping. This trend amounts to an increasingly anisotropic response of the drift atoms to field inhomogeneities parallel and transverse to $\mathbf{B}$. We have performed calculations with different field curvatures, and have confirmed that the effective moments $\mu_{z}$ and $\mu_{\rho}$ shown in Figure 3 are independent of the field curvature.

\section{Quantum mechanical calculation}

For the case $M_{\mathrm{i}}=\infty$, which is a good approximation for rubidium, we have computed magnetic dipole moments from quantum spectra. The moments follow from the energy-level shifts of Rydberg states calculated for two slightly different homogeneous magnetic fields. The magnetic moment of a state that shifts in energy by an amount $\Delta E$ if the magnetic field is changed by a small amount $\Delta B$ is given by $\mu=-\Delta E / \Delta B$.

To calculate the energy levels of bound drift Rydberg states in strong magnetic fields an adiabatic model can be used, as explained in references [21,22]. While exact energy levels can also be easily obtained by accounting for non-adiabatic corrections [22], we find that the magnetic moments derived from adiabatic and exact energy levels are very similar. Therefore, for simplicity we only show magnetic moments obtained from adiabatic energy levels. The results for magnetic quantum numbers $m=-100$ and $m=-250$ are plotted in Figure 4 vs. the energy of the quantum Rydberg levels. Inspection of the figure shows that the data points follow a regular pattern characterized by diagonal lines from the upper left to the lower right. It turns out that each line corresponds to a certain number of energy quanta in the transverse (cyclotron) motion, while the ordering of the data points within each line reflects the number of excitations in the $z$-bounce motion. We denote the respective quantum numbers $n_{\mathrm{c}}$ and $n_{z}$ (both start counting from zero) and indicate them in Figure 4. The figure shows that atoms with larger cyclotron $\left(n_{\mathrm{c}}\right)$ and $z$-bounce $\left(n_{z}\right)$ quantum numbers have more negative magnetic moments, as expected.

Magnetic moments extracted from Figure 4 and corresponding data sets for other $m$-values (not shown) are compared with the classical results in Figure 2. For the
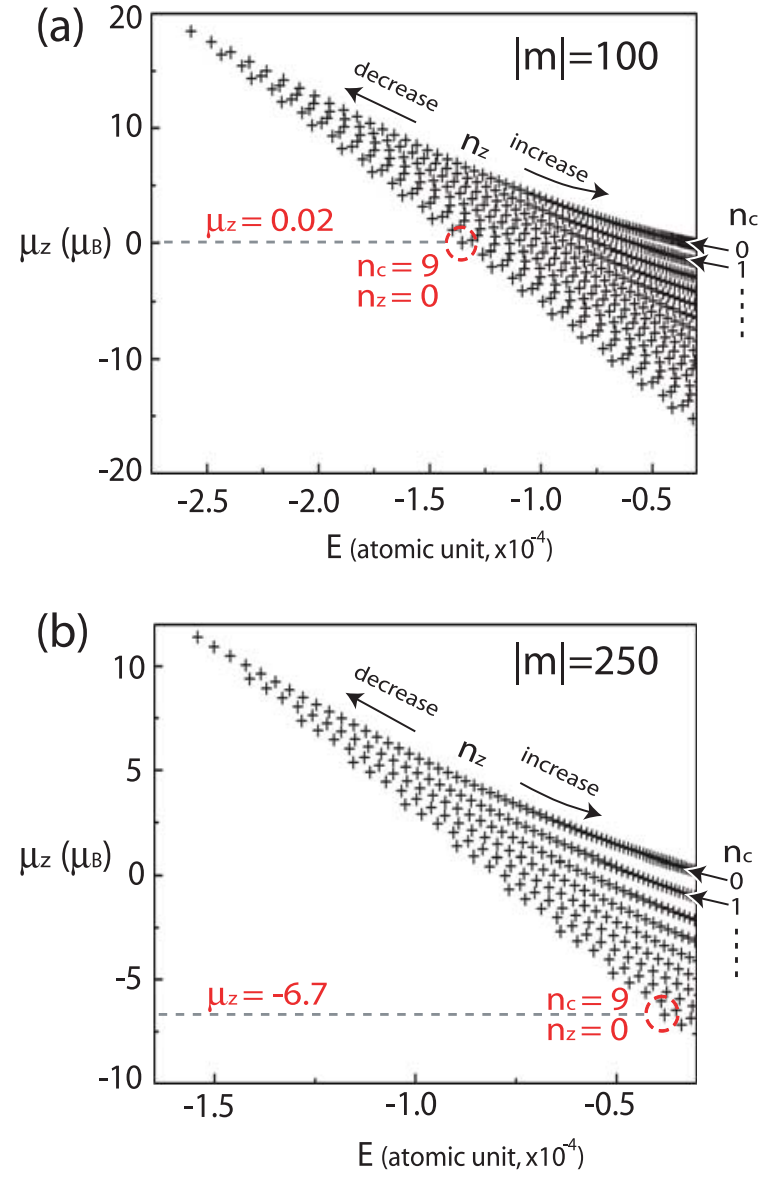

Fig. 4. $\mu_{z}$ for Rydberg states with $m=-100$ (a) and $m=-250$ (b) from quantum-mechanical calculations. Each point corresponds to an adiabatic Rydberg level with specific cyclotron $\left(n_{\mathrm{c}}\right)$ and $z$-bounce $\left(n_{z}\right)$ quantum numbers. The states with $n_{\mathrm{c}}=9$ and $n_{z}=0$, marked in both panels, correspond closely to data in Figure 2 a at $m=-100$ and $m=-250$. A colour version of the figure is available in electronic form at http://www . eurphysj . org.

comparison, we choose quantum levels with $n_{\mathrm{c}}=9$ in order to match the kinetic energy of the perpendicular motion used in Figure $2\left(E_{\text {cyc }}=1.2 \times 10^{-4}\right)$. To obtain quantum analogues of the data in Figure 2a with planar electronic motions, we take the lowest two $n_{z}$-states in the $n_{\mathrm{c}}=9$-series and extrapolate the magnetic moments of these states in a manner that a value corresponding to zero $z$-bounce energy is obtained. As seen in Figure 2a, the agreement between classical and quantum data is excellent. In a similar manner, magnetic moments that correspond to a longitudinal energy $E_{\|}=2 \times 10^{-5}$ can be extracted from Figure 4 and similar plots using an interpolation method between data points. The results, shown in Figure 2b, also agree very well with classical values. The observed agreement is expected based on the correspondence principle, and it justifies our use of classical models to determine the magnetic moments of drift Rydberg states. 


\section{State-analysis of trapped Rydberg atoms}

In the remainder of this paper, we utilize the obtained results to develop an approximate idea of the nature of drift Rydberg states that have been trapped in recent experiments [12]. There, we have found that drift-state Rydberg atoms can be trapped in shallow Ioffe-Pritchard magnetic traps [27] with central magnetic fields of about $3 \mathrm{~T}$ and a trap depth of $\approx 0.03 \mathrm{~T}$. The average magnetic moment of the trapped Rydberg atoms was measured to be $-8 \mu_{\mathrm{B}}$. Here, we characterize the range of states with magnetic moments of that order. These are the states that likely were populated in the high- $B$ Rydberg-atom trapping experiment described in reference [12].

To fill the high- $B$ Ioffe-Pritchard trap with drift Rydberg atoms, we first laser-excite cold atoms prepared in the trap into low- $|m|$ Rydberg states. In the following tens of microseconds, these atoms undergo collisions with free electrons and with other Rydberg atoms, as is also observed under magnetic-field-free conditions [4-8]. In high $B$-fields, Rydberg-Rydberg collisions may be more prevalent than under field-free conditions, because Rydberg atoms in high $B$-fields can have very large permanent quadrupole moments that can cause attractive forces between atoms. Penning-ionizing collisions $[4,8,28]$ generate free electrons with energies in the range of $10 \mathrm{meV}$, which may further collide with other Rydberg atoms. In strong magnetic fields, collisions between Rydberg atoms with free electrons and other Rydberg atoms cause $m$-mixing and partial transformation of the population into high- $|m|$ drift Rydberg states. Since the density of states of the drift atoms is very large, the Rydberg atoms mostly evolve from low- $|m|$ into drift states (with negligible backward evolution). Also, due to the rapid drop of Rydberg-atom and charged-particle density after the laser excitation, the collisions will largely cease after of order 100 microseconds, leaving behind a cloud of drift-state atoms in a distribution of $m$. Further investigations will be required in order to understand this collision-induced evolution in more detail. It is presently not clear whether it will be possible to obtain some control over the $m$-distribution by varying parameters such as the initial density, the Rydberg state, bias static electric fields, electric-field pulses etc.

It is assumed that during the collisions which turn a low- $|m|$ state into a drift-state atom the Rydberg-atom energy does not change very much. In the following classical computation, we therefore consider drift-atom trajectories with a fixed total energy of $E_{\text {tot }}=-3.0 \times 10^{-5}$, which was the laser excitation energy used in reference [12]. The cyclotron energy of the electron, $E_{\text {cyc }}$, is expressed in terms of cyclotron quanta, $n_{\mathrm{c}}=E_{\mathrm{cyc}} / B-1 / 2$ (which in a classical computation is not necessarily an integer). The drift trajectory is further characterized by the $z$-component of the canonical angular momentum, $|m|(m<0)$. The relation $|m|=(B / 2) \rho_{\mathrm{e}}^{2}$ fixes the value of $\rho_{\mathrm{e}}$. The $z$-bounce motion is fully characterized by the value of $\rho_{\mathrm{e}}$ and the longitudinal energy, $E_{\|}=E_{\text {tot }}-E_{\text {cyc }}$. Consequently, the drift trajectories for given total energy $E_{\text {tot }}$ can be represented on the $\left(|m|, n_{\mathrm{c}}\right)$-plane. Their magnetic moments are obtained using equation (8). In order to conform with the

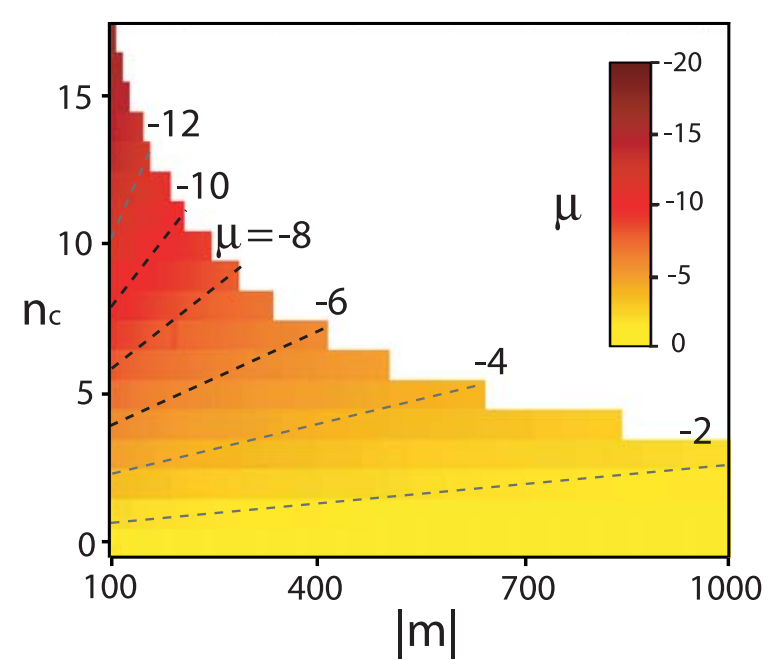

Fig. 5. Magnetic moments of Rb drift-state atoms in units of $\mu_{\mathrm{B}}$ vs. magnetic quantum number $|m|(m<0)$ and cyclotron excitation number $n_{\mathrm{c}}$ obtained from classical calculations. A total energy of $-3.0 \times 10^{-5}$ is assumed, which is equivalent to $n \approx 130$ in $B$-field-free atoms. The total energy is equivalent to the laser-excitation energy of atoms in reference [12]. A colour version of the figure is available in electronic form at http://www. eurphysj.org.

experiment described in [12], which has been performed with a spin-polarized atomic sample, we add a magnetic moment of $-\mu_{\mathrm{B}}$ to account for the intrinsic electron spin. The result in Figure 5 shows that all the drift atoms on the $\left(|m|, n_{\mathrm{c}}\right)$-plane plane would be magnetically trapped (i.e. $\mu<0$ ). Further, the experimentally observed range of magnetic moments, $-11 \mu_{\mathrm{B}} \lesssim \mu \lesssim-5 \mu_{\mathrm{B}}$, is seen to correspond to an $m$ range of $|m| \lesssim 400$ and a $n_{\text {c-range of }}$ $3 \lesssim n_{\mathrm{c}} \lesssim 12$.

As Figure 5 shows, for suitable excitation energy almost all drift-state atoms are magnetically trapped. Once atoms are scattered into trapped states $(\mu<0)$ with $|m|$ of order several hundreds, they have fairly low radiative decay rates [26]. Further, secondary collisions are expected to not change their drift-state character very much. The atoms will tend to remain in trapped states even if the detailed quantum numbers of the atoms might change due to secondary collisions. We believe that for these reasons the experimental demonstration of Rydberg-atom trapping [12] succeeded first in a high-magnetic-field trap.

\section{Summary}

We have investigated the magnetic trapping dynamics of drift Rydberg states by computing magnetic moments both classically and quantum mechanically. In $\mathrm{Rb}$ drift Rydberg atoms, the magnetic moment can be attributed to the cyclotron and the magnetron motion of the Rydberg electron. In the case of hydrogen, the motion of the ion needs to be considered additionally, revealing the twobody nature of the problem. We have found an anisotropic response of hydrogen drift atoms to magnetic-field inhomogeneities longitudinal and transverse to $\mathbf{B}$. The 
anisotropy is expected to limit the range of drift states that could be magnetically trapped in antihydrogen experiments. The results have been applied to estimate the range of states that have been trapped in a recent Rydberg-atom trapping experiment. Radiative decay of drift Rydberg atoms has not been considered in the stateanalysis presented in this paper. Generally, states with higher $n_{\mathrm{c}}$ or lower $|m|$ have smaller lifetimes than the states with lower $n_{\mathrm{c}}$ or higher $|m|$ [26]. Therefore, measurements of the average lifetime of trapped drift Rydberg atoms could, in future, be used for further analysis of the trapped atoms.

This work was supported by the Chemical Sciences, Geosciences and Biosciences Division of the Office of Basic Energy Sciences, Office of Science, U.S. Department of Energy.

\section{References}

1. D. Jaksch et al., Phys. Rev. Lett. 85, 2208 (2000)

2. G. Gabrielse et al., Phys. Rev. Lett. 89, 213401 (2002)

3. S.K. Dutta, J.R. Guest, D. Feldbaum, A. Walz-Flannigan, G. Raithel, Phys. Rev. Lett. 85, 5551 (2000)

4. M.P. Robinson, B. Laburthe Tolra, M.W. Noel, T.F. Gallagher, P. Pillet, Phys. Rev. Lett. 85, 4466 (2000)

5. S.K. Dutta, D. Feldbaum, A. Walz-Flannigan, J.R. Guest, G. Raithel, Phys. Rev. Lett. 86, 3993 (2001)

6. A. Walz-Flannigan, J.R. Guest, J.-H. Choi, G. Raithel, Phys. Rev. A 69, 63405 (2004)

7. W. Li et al., Phys. Rev. A 70, 042713 (2004)

8. W. Li, P.J. Tanner, T.F. Gallagher, Phys. Rev. Lett. 94, $173001(2005)$
9. P. Hyafil et al., Phys. Rev. Lett. 93, 103001 (2004)

10. I. Lesanovsky, P. Schmelcher, Phys. Rev. Lett. 95, 053001 (2005)

11. I. Lesanovsky, P. Schmelcher, Phys. Rev. A 72, 053410 (2005)

12. J.-H. Choi, J.R. Guest, A.P. Povilus, E. Hansis, G. Raithel, Phys. Rev. Lett. 95, 243001 (2005)

13. M.E. Glinsky, T.M. O’Neil, Phys. Fluids B 3, 1279 (1991)

14. M. Amoretti et al., Nature 419, 456 (2002)

15. G. Gabrielse et al., Phys. Rev. Lett. 89, 213401 (2002)

16. G. Gabrielse et al., Phys. Rev. Lett. 89, 233401 (2002)

17. S.G. Kuzmin, T.M. O'Neil, Phys. Rev. Lett. 92, 243401 (2004)

18. S.G. Kuzmin, T.M. O'Neil, Phys. Plasmas 12, 12101 (2005)

19. G. Raithel, M. Fauth, J. Phys. B 28, 1687 (1995)

20. L.D. Landau, E.M. Lifshitz, Quantum Mechanics: NonRelativistic Theory, 3rd edn. (Pergamon Press, 1977)

21. Q. Wang, C.H. Greene, Phys. Rev. A 40, 742 (1989)

22. J.R. Guest, G. Raithel, Phys. Rev. A 68, 052502 (2003)

23. J.-H. Choi, J.R. Guest, E. Hansis, A.P. Povilus, G. Raithel, Phys. Rev. Lett. 95, 253005 (2005)

24. J.D. Jackson, Classical Electrodynamics, 3rd edn. (John Wiley \& Sons, Inc., 1999)

25. B.R. Johnson, J.O. Hirschfelder, Kuo-Ho Yang, Rev. Mod. Phys. 55, 109 (1983)

26. J.R. Guest, J.-H. Choi, G. Raithel, Phys. Rev. A 68, $022509(2003)$

27. J.R. Guest, J.-H. Choi, E. Hansis, A.P. Povilus, G. Raithel, Phys. Rev. Lett. 94, 073003 (2005)

28. B. Knuffman, G. Raithel, Phys. Rev. A 73, 020704(R) (2006) 